\title{
Solitary Wave Solutions and Rational Solutions for Modified Zakharov-Kuznetsov Equation with Initial Value Problem
}

\author{
Zhongzhou Dong1 ${ }^{*}$, Ling Wang2 \\ ${ }^{1}$ School of Mathematics and Information Science, Henan Polytechnic University, Jiaozuo, China \\ ${ }^{2}$ Academic Administration, Henan Polytechnic University, Jiaozuo, China \\ Email: zzdong@hpu.edu.cn
}

How to cite this paper: Dong, Z.Z. and Wang, L. (2018) Solitary Wave Solutions and Rational Solutions for Modified Zakharov-Kuznetsov Equation with Initial Value Problem. Journal of Applied Mathematics and Physics, 6, 949-959. https://doi.org/10.4236/jamp.2018.65081

Received: March 8, 2018

Accepted: May 12, 2018

Published: May 15, 2018

Copyright ( $) 2018$ by authors and Scientific Research Publishing Inc. This work is licensed under the Creative Commons Attribution International License (CC BY 4.0).

http://creativecommons.org/licenses/by/4.0/

\begin{abstract}
The modified Zakharov-Kuznetsov equation with the initial value problem is studied numerically by means of homotopy perturbation method. The analytical approximate solutions of the modified Zakharov-Kuznetsov equation are obtained. Choosing the form of the initial value, the single solitary wave, two solitary waves and rational solutions are presented, some of which are shown by the plots.
\end{abstract}

\section{Keywords}

Modified Zakharov-Kuznetsov Equation, Homotopy Perturbation Method, Soliton Solution

\section{Introduction}

Partial differential equations widely describe many phenomena in the world. Although many mathematicians and physicists presented various methods to find the explicit solutions of the partial differential equations, it is a difficult and important task to build the solutions of initial and boundary value problem. Recently, homotopy perturbation method (HPM) has been applied into many problems [1]-[10] and tested to be an effective tool. Here, the initial value problem of the modified Zakharov-Kuznetsov (mZK) equation is studied by using HPM.

The initial value problem of $\mathrm{mZK}$ equation is as following:

$$
\left\{\begin{array}{l}
u_{t}+u^{2} u_{x}+u_{x x x}+u_{x y y}=0, \\
u(x, y, 0)=f(x, y) .
\end{array}\right.
$$


Many authors have studied the mZK equation [11]-[16]. The authors in Ref. [11] applied the asymptotic approach into $\mathrm{mZK}$ equation and found for the $\mathrm{mZK}$ equation that critical collapse in two dimensions is accompanied by damping both the momentum and energy of the perturbed solitary waves and that slows down the rate of the singularity formation. Ref. [12] considered the two-dimensional solitary wave (lump) interactions and the formation of singularities in mZK equation. Ref. [13] obtained a class of approximate periodic solutions for mZK equation by using the homotopy analysis method. The authors in Ref. [14] obtained many solitary waves and periodic waves and kink waves of mZK equation by using the theory of bifurcations of dynamical systems. Uisng the extended tanh method, Ref. [15] got new travelling wave solutions with solitons and periodic structures. In Ref. [16], the first integral method was used to construct travelling wave solutions of mZK equation. Peng [17] developed the extended mapping method to study the traveling wave solution for the mZK equation. Ref. [18] applied the exp-function method to construct generalized solitary and periodic solutions of mZK equation. Authors in Ref. [19] applied the improved $\left(\mathrm{G}^{\prime} / \mathrm{G}\right)$-expansion method to construct abundant new exact traveling wave solutions of mZK equation. Ref. [20] employed the complex method to obtain the exact solutions of mZK equation.

This paper is arranged as follows: In Section 2, by using HPM, we obtain the analytical approximate solution of Equation (1). By taking the form of the initial value, some exact solutions of mZK equation are obtained in Section 3. And some pictures are given to show the structure of the obtained solutions. Finally, some conclusions and discussions are given in Section 4.

\section{The Homotopy Perturbation Method to mZK Equation}

In order to obtain the analytical approximate solution of Equation (1), we consider the one-parameter family of Equation (1) as follows

$$
\left(u-u_{0}\right)_{t}+p\left(u^{2} u_{x}+u_{x x x}+u_{x y y}\right)=0,
$$

where the parameter $p \in[0,1]$ and $u_{0}=f(x, y)$.

If $p=0$, we meet $u=u_{0}$.

If $p=1$, we come back to the original problem (1). Let the solution $u(x, y, t)$ of the system (2) be written in the form of an infinite series,

$$
u(x, y, t)=\sum_{i=0}^{\infty} u_{i}(x, y, t) p^{i} .
$$

Then $u(x, y, t)=\sum_{i=0}^{\infty} u_{i}(x, y, t)$ is a series solution of Equation (1).

Substituting Equation (3) into Equation (2), and equating the coefficients of $p, p^{2}, \cdots$, we have

$$
\begin{gathered}
u_{1, t}+u_{0}^{2} u_{0, x}+u_{0, x x x}+u_{0, x y y}=0, \\
u_{2, t}+u_{0}^{2} u_{1, x}+2 u_{0} u_{1} u_{0, x}+u_{1, x x x}+u_{1, x y y}=0, \\
u_{3, t}+u_{0}^{2} u_{2, x}+u_{1}^{2} u_{0, x}+2 u_{0} u_{1} u_{1, x}+2 u_{0} u_{2} u_{0, x}+u_{2, x x x}+u_{2, x y y}=0,
\end{gathered}
$$


and so on. Solving Equation (4), (5) and (6), one can obtain

$$
\begin{aligned}
& u_{1}(x, y, t)=-\left(u_{0}^{2} u_{0, x}+u_{0, x x x}+u_{0, x y y}\right) t, \\
& u_{2}(x, y, t)=\frac{1}{2}\left(4 u_{0}^{3} u_{0, x}^{2}+u_{0}^{4} u_{0, x x}+2 u_{0}^{2} u_{0, x x x x}+2 u_{0}^{2} u_{0, x x y y}+12 u_{0, x}^{2} u_{0, x x}\right. \\
& +6 u_{0} u_{0, x x}^{2}+10 u_{0} u_{0, x} u_{0, x x x}+2 u_{0, x}^{2} u_{0, y y}+8 u_{0, x} u_{0, y} u_{0, x y} \\
& +4 u_{0} u_{0, x y}^{2}+6 u_{0} u_{0, x} u_{0, x y y}+2 u_{0, y}^{2} u_{0, x x}+4 u_{0} u_{0, y} u_{0, x x y} \\
& \left.+2 u_{0} u_{0, x x} u_{0, y y}+u_{0, x x x x x x}+2 u_{0, x x x x y y}+u_{0, x x y y y y}\right) t^{2} \text {, } \\
& u_{3}(x, y, t)=-\frac{1}{6}\left(30 u_{0} u_{0, x y y} u_{0, x x x x}+66 u_{0, x x}^{2} u_{0, x y y}+76 u_{0} u_{0, x} u_{0, y}^{2} u_{0, x x}\right. \\
& +92 u_{0}^{2} u_{0, x} u_{0, x x y} u_{0, x x x}+84 u_{0}^{2} u_{0, y} u_{0, x x} u_{0, x y}+46 u_{0}^{2} u_{0, x} u_{0, x x} u_{0, y y} \\
& +160 u_{0} u_{0, x}^{2} u_{0, y} u_{0, x y}+3 u_{0}^{2} u_{0, x x x x x x}+3 u_{0}^{4} u_{0, x x x y y}+216 u_{0, x x}^{2} u_{0, x x x} \\
& +150 u_{0, x} u_{0, x x x}^{2}+12 u_{0} u_{0, y} u_{0, x x x y y y}+46 u_{0} u_{0, x y y} u_{0, x x y y} \\
& +66 u_{0}^{3} u_{0, x x} u_{0, x x x}+3 u_{0}^{4} u_{0, x x x x x}+174 u_{0}^{2} u_{0, x}^{2} u_{0, x x x}+18 u_{0}^{5} u_{0, x} u_{0, x x} \\
& +84 u_{0} u_{0, x x x} u_{0, x x x x}+168 u_{0, x} u_{0, x y} u_{0, x x x y}+12 u_{0} u_{0, y} u_{0, x x x x x y} \\
& +84 u_{0, y} u_{0, x x x} u_{0, x x y}+84 u_{0, y} u_{0, x x} u_{0, x x x y}+48 u_{0, x} u_{0, y} u_{0, x x x x y} \\
& +42 u_{0}^{3} u_{0, x} u_{0, x x x x}+264 u_{0} u_{0, x}^{3} u_{0, x x}+24 u_{0, x} u_{0, y y} u_{0, x x x x}+u_{0}^{6} u_{0, x x x} \\
& +8 u_{0} u_{0, y y y} u_{0, x x x y}+30 u_{0}^{4} u_{0, x}^{3}+56 u_{0, x} u_{0, x y} u_{0, x y y y}+84 u_{0} u_{0, x x y} u_{0, x x x y} \\
& +6 u_{0} u_{0, y y} u_{0, x x x x x}+14 u_{0}^{2} u_{0, y}^{2} u_{0, x x x}+24 u_{0, y} u_{0, y y} u_{0, x x x y}+8 u_{0, x}^{2} u_{0, x y y y y} \\
& +14 u_{0} u_{0, y y} u_{0, x x x y y}+40 u_{0, x} u_{0, y y} u_{0, x x y y}+72 u_{0, x y} u_{0, y y} u_{0, x x y} \\
& +6 u_{0, x} u_{0, x x} u_{0, y y y y}+24 u_{0, x x} u_{0, x y} u_{0, y y y}+36 u_{0} u_{0, x} u_{0, x x x x y y} \\
& +54 u_{0} u_{0, x x x} u_{0, x x y y}+24 u_{0}^{3} u_{0, x x} u_{0, x y y}+28 u_{0, y} u_{0, x x} u_{0, x y y y} \\
& +8 u_{0} u_{0, x x} u_{0, x y y y y}+48 u_{0, y} u_{0, x y} u_{0, x x x x}+28 u_{0} u_{0, x x y} u_{0, x y y y} \\
& +54 u_{0} u_{0, x x} u_{0, x x x x x}+24 u_{0} u_{0, x} u_{0, x x x x x x}+54 u_{0} u_{0, x x} u_{0, x x x y y} \\
& +234 u_{0, x} u_{0, x x} u_{0, x x x x}+48 u_{0} u_{0, x y} u_{0, x x x x y}+144 u_{0, x} u_{0, x x} u_{0, x x y y} \\
& +76 u_{0, y} u_{0, x x y} u_{0, x y y}+24 u_{0, x}^{5}+2 u_{0} u_{0, x x x} u_{0, y y y y}+24 u_{0, x}^{3} u_{0, y}^{2} \\
& +80 u_{0, y} u_{0, x y} u_{0, x x y y}+32 u_{0} u_{0, x y} u_{0, x x y y y}+32 u_{0, x} u_{0, y} u_{0, x x y y y} \\
& +12 u_{0} u_{0, x} u_{0, x x y y y y}+28 u_{0} u_{0, x}^{3} u_{0, y y}+38 u_{0, x x} u_{0, y y} u_{0, x y y} \\
& +42 u_{0, x x} u_{0, y y} u_{0, x x x}+24 u_{0, x} u_{0, x x y} u_{0, y y y}+240 u_{0, x x} u_{0, x y} u_{0, x x y} \\
& +30 u_{0}^{3} u_{0, x} u_{0, x x y y}+222 u_{0}^{2} u_{0, x} u_{0, x x}^{2}+92 u_{0}^{2} u_{0, x} u_{0, x y}^{2}+6 u_{0}^{3} u_{0, y y} u_{0, x x x} \\
& +36 u_{0}^{3} u_{0, x y} u_{0, x x y}+6 u_{0}^{2} u_{0, x x x x x y y}+12 u_{0}^{3} u_{0, y} u_{0, x x x y}+68 u_{0}^{2} u_{0, x}^{2} u_{0, x y y} \\
& +96 u_{0, x} u_{0, x x x} u_{0, x y y}+8 u_{0, y} u_{0, x x x} u_{0, y y y}+6 u_{0, y y}^{2} u_{0, x x x}+42 u_{0, x} u_{0, x y y}^{2} \\
& +14 u_{0, y}^{2} u_{0, x x x y y}+76 u_{0, x y}^{2} u_{0, x y y}+54 u_{0, x}^{2} u_{0, x x x x x}+6 u_{0, y}^{2} u_{0, x x x x x} \\
& +84 u_{0, x y}^{2} u_{0, x x x}+54 u_{0, x}^{2} u_{0, x x x y y}+120 u_{0, x} u_{0, x x y}^{2}+3 u_{0}^{2} u_{0, x x x y y y} \\
& \left.+u_{0, x x x x x x x x x}+3 u_{0, x x x x x x x y y}+3 u_{0, x x x x x y y y y}+u_{0, x x x y y y y y y}\right) t^{3} .
\end{aligned}
$$

Hence, we obtain the solution of Equation (1)

$$
u(x, y, t)=f(x, y)+u_{1}(x, y, t)+u_{2}(x, y, t)+u_{3}(x, y, t)+\cdots,
$$


where $u_{1}, u_{2}$ and $u_{3}$ are given by Equation (7), (8) and (9) respectively.

\section{Application}

In this section, we will study the single soliton, two-soliton and rational solutions of mZK equation.

\subsection{Single Solitary Wave Solution}

Consider the following case:

$$
\left\{\begin{array}{l}
u_{t}+u^{2} u_{x}+u_{x x x}+u_{x y y}=0, \\
u(x, y, 0)=\frac{2 k \sqrt{6\left(1+m^{2}\right)} \exp (k(x+m y))}{\exp (2 k(x+m y))+1} .
\end{array}\right.
$$

From the above section, we can have

$$
\begin{gathered}
u_{0}(x, y, t)=\frac{2 k \sqrt{6\left(1+m^{2}\right)} \exp (k(x+m y))}{\exp (2 k(x+m y))+1}, \\
u_{1}(x, y, t)=\frac{2 k^{4}\left(1+m^{2}\right) \sqrt{6\left(1+m^{2}\right)} \exp (k(x+m y))}{(\exp (2 k(x+m y))+1)^{2}}(\exp (2 k(x+m y))-1) t, \\
u_{2}(x, y, t)=\frac{k^{7}\left(1+m^{2}\right)^{2} \sqrt{6\left(1+m^{2}\right)} \exp (k(x+m y))}{(\exp (2 k(x+m y))+1)^{3}} \\
u_{3}(x, y, t)=\frac{k^{10}\left(1+m^{2}\right)^{3} \sqrt{6\left(1+m^{2}\right)} \exp (k(x+m y))}{3(\exp (2 k(x+m y))+1)^{4}}(\exp (6 k(x+m y)) \\
-23 \exp (4 k(x+m y))+23 \exp (2 k(x+m y))-1) t^{3}, \\
x(x, y, t)=\frac{2 k \sqrt{6\left(1+m^{2}\right)} \exp (k(x+m y))}{\exp (2 k(x+m y))+1} \\
\times(\exp (6 k(x+m y))-23 \exp (4 k(x+m y))+23 \exp (2 k(x+m y))-1) t^{3}+\cdots . \\
+\frac{2 k^{4}\left(1+m^{2}\right) \sqrt{6\left(1+m^{2}\right)} \exp (k(x+m y))}{(\exp (2 k(x+m y))+1)^{2}}(\exp (2 k(x+m y))-1) t \\
+\frac{(\exp (2 k(x+m y))+1)^{3}}{k^{7}\left(1+m^{2}\right)^{2} \sqrt{6\left(1+m^{2}\right)} \exp (k(x+m y))}(\exp (4 k(x+m y)) \\
k^{10}\left(1+m^{2}\right)^{3} \sqrt{6\left(1+m^{2}\right)} \exp (k(x+m y)) \\
+\exp (2 k(x+m y))+1)^{4}
\end{gathered}
$$

Using Taylor series, one can obtain the exact solution 


$$
u(x, y, t)=\frac{2 k \sqrt{6\left(1+m^{2}\right)} \exp \left(k\left(x+m y-k^{2}\left(1+m^{2}\right) t\right)\right)}{\exp \left(2 k\left(x+m y-k^{2}\left(1+m^{2}\right) t\right)\right)+1} .
$$

Figure 1 shows the single soliton (17) for $k=1, m=1,-4 \leq x \leq 4$, $-4 \leq y \leq 4$ and $t=0$, from which one can find Equation (17) is a single-soliton solution.

\subsection{Two Solitary Waves Solution}

In this case, we take

$$
f(x, y)=\frac{4 \sqrt{6\left(1+m^{2}\right)} \exp (x+m y)}{\exp (2(x+m y))+1}
$$

Then from the above section, one can have

$$
\begin{aligned}
& u_{0}(x, y, t)=\frac{4 \sqrt{6\left(1+m^{2}\right)} \exp (x+m y)}{\exp (2(x+m y))+1}, \\
u_{1}(x, y, t)= & \frac{4\left(1+m^{2}\right) \sqrt{6\left(1+m^{2}\right)} \exp (x+m y)}{(\exp (2(x+m y))+1)^{4}}(\exp (6(x+m y)) \\
+ & 73 \exp (4(x+m y))-73 \exp (2(x+m y))-1) t, \\
u_{2}(x, y, t)= & \frac{2\left(1+m^{2}\right)^{2} \sqrt{6\left(1+m^{2}\right)} \exp (x+m y)}{(\exp (2(x+m y))+1)^{7}}(\exp (12(x+m y)) \\
& +2158 \exp (10(x+m y))+2863 \exp (8(x+m y)) \\
& -26236 \exp (6(x+m y))+2863 \exp (4(x+m y)) \\
& +2158 \exp (2(x+m y))+1) t^{2}
\end{aligned}
$$

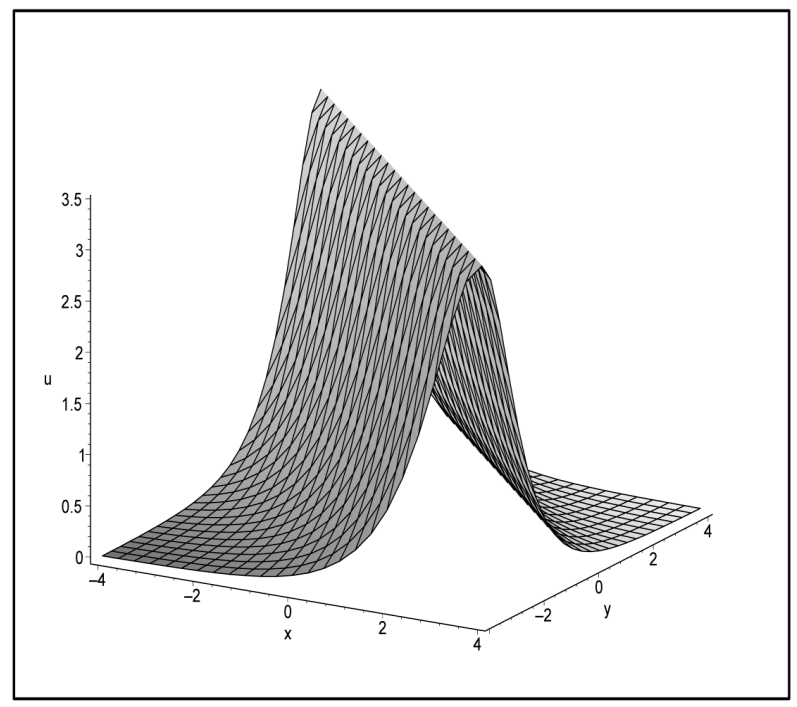

Figure 1. 3D plot of solution (17) for $k=-1$. 


$$
\begin{aligned}
& u_{3}(x, y, t)=\frac{2\left(1+m^{2}\right)^{3} \sqrt{6\left(1+m^{2}\right)} \exp (x+m y)}{3(\exp (2(x+m y))+1)^{10}}(\exp (18(x+m y)) \\
& +58951 \exp (16(x+m y))+225620 \exp (14(x+m y)) \\
& -1999268 \exp (12(x+m y))-6147250 \exp (10(x+m y)) \\
& +6147250 \exp (8(x+m y))+1999268 \exp (6(x+m y)) \\
& -225620 \exp (4(x+m y))-58951 \exp (2(x+m y))-1) t^{3}, \\
& u(x, y, t)=\frac{4 \sqrt{6\left(1+m^{2}\right)} \exp (x+m y)}{\exp (2(x+m y))+1}+\frac{4\left(1+m^{2}\right) \sqrt{6\left(1+m^{2}\right)} \exp (x+m y)}{(\exp (2(x+m y))+1)^{4}} \\
& \times(\exp (6(x+m y))+73 \exp (4(x+m y))-73 \exp (2(x+m y))-1) t \\
& +\frac{2\left(1+m^{2}\right)^{2} \sqrt{6\left(1+m^{2}\right)} \exp (x+m y)}{(\exp (2(x+m y))+1)^{7}}(\exp (12(x+m y)) \\
& +2158 \exp (10(x+m y))+2863 \exp (8(x+m y))-26236 \exp (6(x+m y)) \\
& +2863 \exp (4(x+m y))+2158 \exp (2(x+m y))+1) t^{2} \\
& +\frac{2\left(1+m^{2}\right)^{3} \sqrt{6\left(1+m^{2}\right)} \exp (x+m y)}{3(\exp (2(x+m y))+1)^{10}}(\exp (18(x+m y)) \\
& +58951 \exp (16(x+m y))+225620 \exp (14(x+m y)) \\
& -1999268 \exp (12(x+m y))-6147250 \exp (10(x+m y)) \\
& +6147250 \exp (8(x+m y))+1999268 \exp (6(x+m y)) \\
& -225620 \exp (4(x+m y))-58951 \exp (2(x+m y))-1) t^{3}+\cdots .
\end{aligned}
$$

Using Taylor series, one can obtain the exact solution

$$
u(x, y, t)
$$$$
=\frac{4 \sqrt{6\left(1+m^{2}\right)}(\exp (\xi-\eta)+3 \exp (27 \xi-3 \eta)+3 \exp (29 \xi-5 \eta)+\exp (55 \xi-7 \eta))}{1+4 \exp (2 \xi-2 \eta)+6 \exp (28 \xi-4 \eta)+4 \exp (54 \xi-6 \eta)+\exp (56 \xi-8 \eta)}
$$

where $\xi=\left(1+m^{2}\right) t$ and $\eta=x+m y$.

Figure 2 shows the two-soliton solution (24) for $m=1,-10 \leq x \leq 5$, $-10 \leq y \leq 5$ and $t=-0.2$, from which one can find Equation (24) is a two-soliton solution. Figure 3 shows the two-soliton solution (24) for $m=1$, $-8 \leq x \leq 8 \quad-8 \leq y \leq 8$ and $t=-0.1$. Figure 4 shows the two-soliton solution (24) for $m=1,-10 \leq x \leq 10-10 \leq y \leq 10$ and $t=0$. Figure 5 shows the two-soliton solution (24) for $m=1,-8 \leq x \leq 8 \quad-8 \leq y \leq 8$ and $t=0.1$. Figure 6 shows the two-soliton solution (24) for $m=1,-5 \leq x \leq 10-5 \leq y \leq 10$ and $t=0.2$. Figures $2-6$ show the velocities of the two solitons are different. 


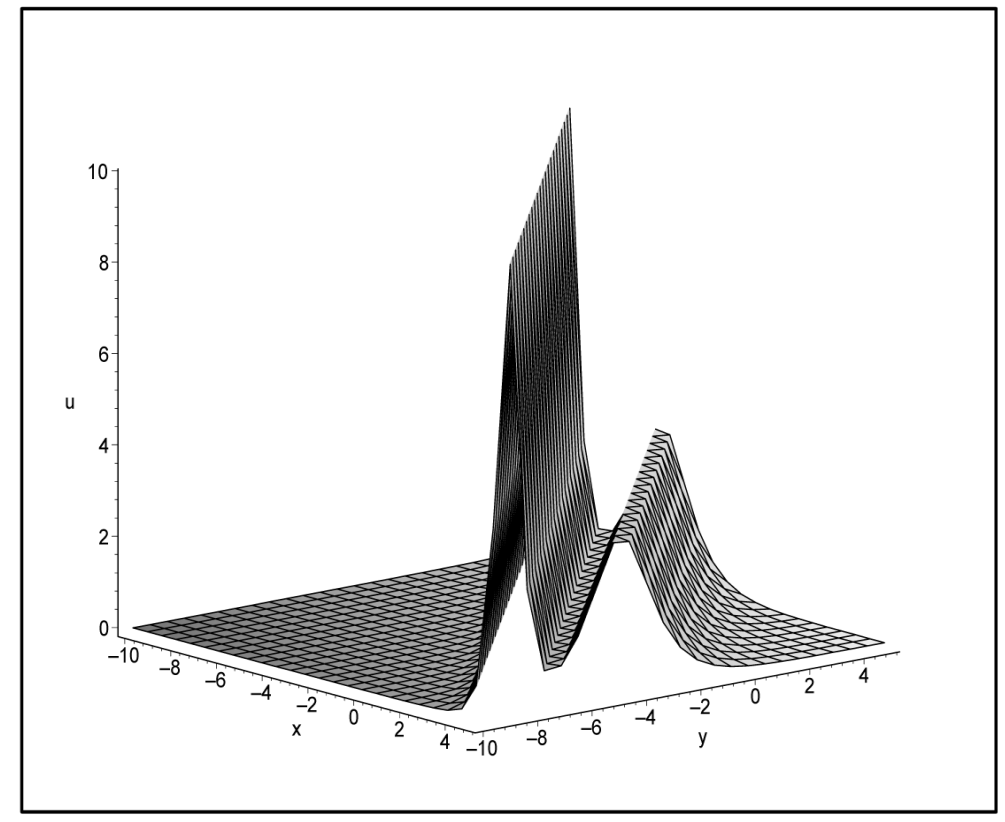

Figure 2. 3D plot of solution (24) for $m=1$ and $t=-0.2$.

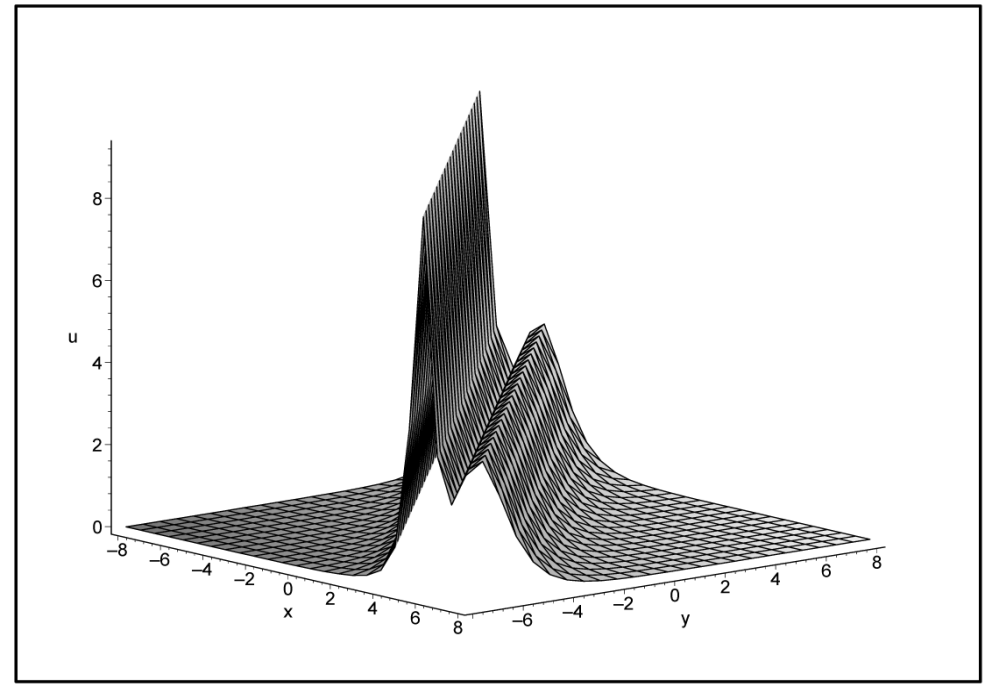

Figure 3. 3D plot of solution (24) for $m=1$ and $t=-0.1$.

\subsection{Rational Solution}

Here, our goal is to find the rational solution of mZK equation. To do this, we consider the form of the initial value as follows:

$$
f(x, y)=\frac{2 I \sqrt{6\left(1+m^{2}\right)}}{x+m y-a} .
$$

Due to the above section, it is obtained

$$
u_{0}(x, y, t)=\frac{2 I \sqrt{6\left(1+m^{2}\right)}}{x+m y-a},
$$




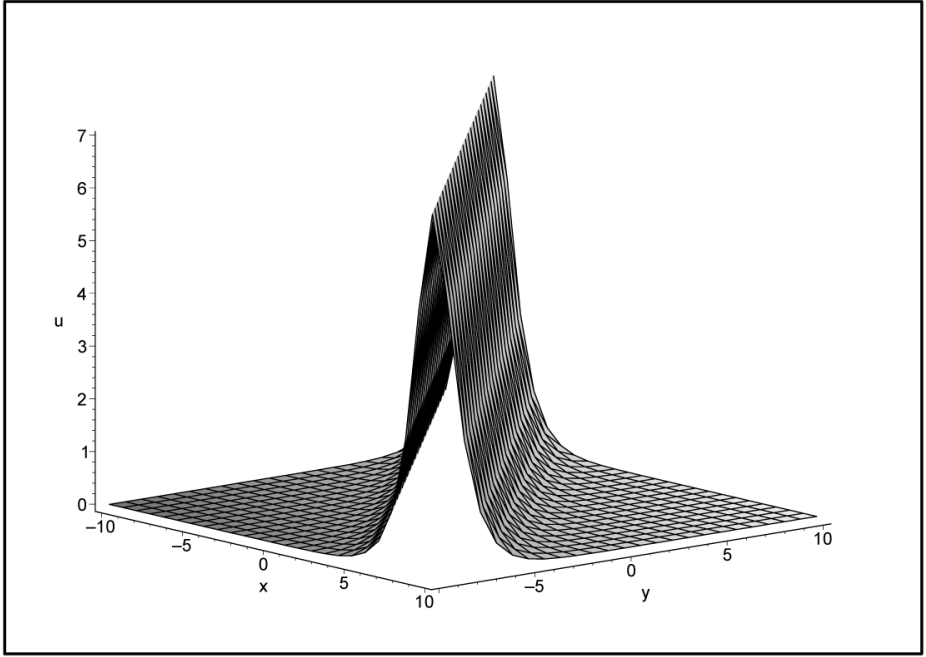

Figure 4. 3D plot of solution (24) for $m=1$ and $t=0$.

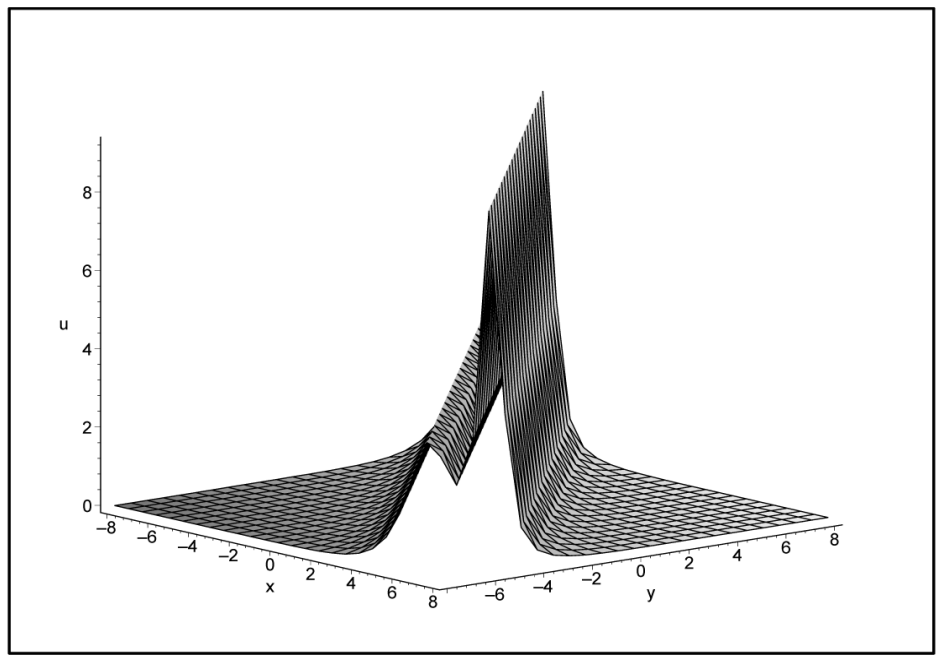

Figure 5. 3D plot of solution (24) for $m=1$ and $t=0.1$.

$$
\begin{gathered}
u_{1}(x, y, t)=-\frac{36 I \sqrt{6\left(1+m^{2}\right)}\left(1+m^{2}\right)}{(x+m y-a)^{4}} t, \\
u_{2}(x, y, t)=\frac{432 I \sqrt{6\left(1+m^{2}\right)}\left(1+m^{2}\right)^{2}}{(x+m y-a)^{7}} t^{2}, \\
u(x, y, t)=\frac{2 I \sqrt{6\left(1+m^{2}\right)}}{u_{3}(x, y, t)=-\frac{5184 I \sqrt{6\left(1+m^{2}\right)}\left(1+m^{3}\right)}{(x+m y-a)^{10}} t^{3},} \\
+\frac{\left(x+\frac{m y-a)^{4}}{6\left(1+m^{2}\right)}\left(1+m^{2}\right)\right.}{432 I \sqrt{6\left(1+m^{2}\right)}\left(1+m^{2}\right)^{2}} t^{2}-\frac{5184 I \sqrt{6\left(1+m^{2}\right)}\left(1+m^{3}\right)}{(x+m y-a)^{7}} t^{3}+\cdots .
\end{gathered}
$$




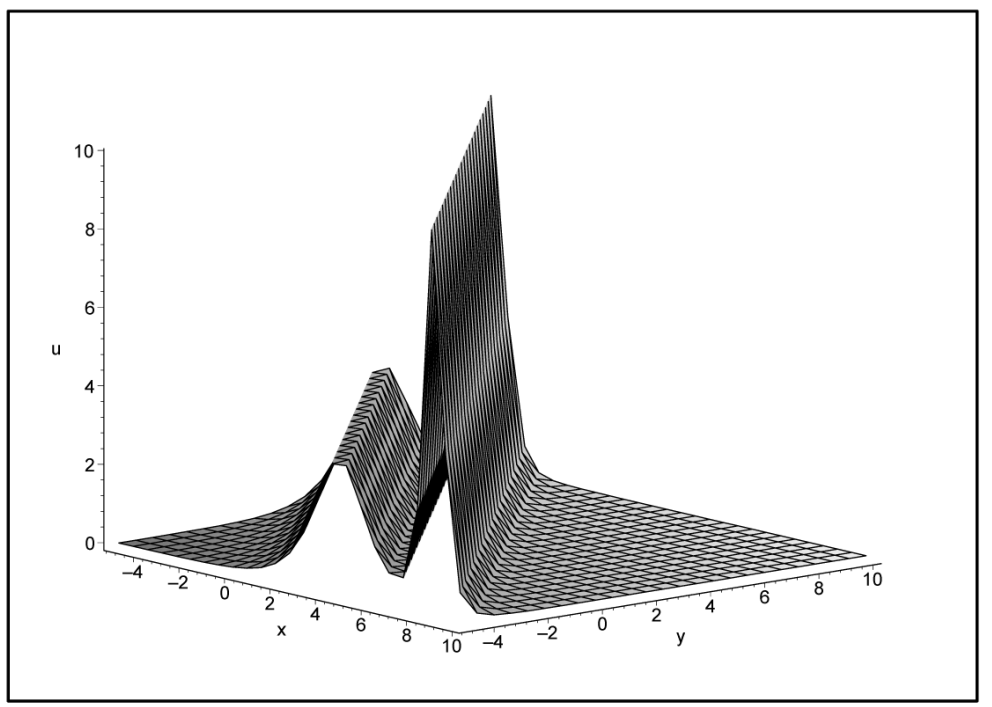

Figure 6. 3D plot of solution (24) for $m=1$ and $t=0.2$.

From the knowledge of Taylor series, one can get the exact solution

$$
u(x, y, t)=\frac{2 I \sqrt{6\left(1+m^{2}\right)}\left((x+m y-a)^{3}-6\left(1+m^{2}\right) t\right)}{(x+m y-a)\left((x+m y-a)^{3}+12\left(1+m^{2}\right) t\right)},
$$

which is singular at $x+m y=a$ or $(x+m y-a)^{3}+12\left(1+m^{2}\right) t=0$.

\section{Conclusion}

In summary, we successfully apply homotopy perturbation method to the mZK equation with the initial value problem and obtain the analytical approximate solution of the $\mathrm{mZK}$ equation. Using the form of the initial value, the single solitary wave, two solitary waves and rational solutions of the mZK are obtained. Here, we get the two-soliton solution without using bilinear forms, Wronskian. In our later works, we will focus on the form of the initial value that can create the two solitary waves solutions.

\section{Acknowledgements}

This work was supported by the National Natural Science Foundation of China under Grant No. 11305048, the Science and Technology Research Key Project of Education Department of Henan Province under Grant No. 13A110329, the Basic and Frontier Research Program of Henan Province under Grant No. 132300410223, the Doctor Foundation of Henan Polytechnic University under Grant No. B2011-006, and the Key Teacher Foundation of Henan Polytechnic University (Grant 2014).

\section{References}

[1] Chow, S.N., Mallet-Paret, J. and Yorke, J.A. (1978) Finding Zeros of Maps: Homotopy Methods That Are Constructive with Probability One. Mathematics of Com- 
putation, 32, 887-899. https://doi.org/10.1090/S0025-5718-1978-0492046-9

[2] Watson, L.T. (1986) Numerical Linear Algebra Aspects of Globally Convergent Homotopy Methods. SIAM Review, 28, 529-545. https://doi.org/10.1137/1028157

[3] Li, T.Y. and Sauser, T. (1987) Homotopy Methods for Eigenvalue Problems. Linear Algebra and its Applications, 91, 65-74. https://doi.org/10.1016/0024-3795(87)90060-7

[4] Liao, S.J. (1995) An Approximate Solution Technique Not Depending on Small Parameters: A Special Example. International Journal of Non-Linear Mechanics, 30, 371-380. https://doi.org/10.1016/0020-7462(94)00054-E

[5] He, J.H. (2004) Comparison of Homotopy Perturbation Method and Homotopy Analysis Method. Applied Mathematics and Computation, 156, 527-539. https://doi.org/10.1016/j.amc.2003.08.008

[6] Ganji, D.D. and Sadighi, A. (2006) Application of He's Homotopy-Perturbation Method to Nonlinear Coupled Systems of Reaction-Diffusion Equations. International Journal of Nonlinear Sciences and Numerical Simulation, 7, 411-418. https://doi.org/10.1515/IJNSNS.2006.7.4.411

[7] Javidi, M. and Golbabai, A. (2007) A Numerical Solution for Solving System of Fredholm Integral Equations by Using Homotopy Perturbation Method. Applied Mathematics and Computation, 189, 1921-1928.

https://doi.org/10.1016/j.amc.2006.12.070

[8] Wang, Q. (2007) Homotopy Perturbation Method for Fractional KdV Equation, Applied Mathematics and Computation, 190, 1795-1802. https://doi.org/10.1016/j.amc.2007.02.065

[9] Odibat, Z. (2007) A New Modification of the Homotopy Perturbation Method for Linear and Nonlinear Operators. Applied Mathematics and Computation, 189, 746-753. https://doi.org/10.1016/j.amc.2006.11.188

[10] Abbasbandy, S. (2007) Application of He's Homotopy Perturbation Method to Functional Integral Equations. Chaos Solitons \& Fractals, 31, 1243-1247. https://doi.org/10.1016/j.chaos.2005.10.069

[11] Pelinovsky, D.E. and Grimshaw, R.H.J. (1996) An Asymptotic Approach to Solitary Wave Instability and Critical Collapse in Long-Wave KdV-Type Evolution Equations. Physica D, 98, 139-155. https://doi.org/10.1016/0167-2789(96)00093-0

[12] Sipcic, R. and Benney, D.J. (2000) Lump Interactions and Collapse in the Modified Zakharov-Kuznetsov Equation. Studies in Applied Mathematics, 105, 385-403. https://doi.org/10.1111/1467-9590.00157

[13] Shi, Y.R., Xu, X.J., Wu, Z.X., Wang, Y.H., Yang, H.J., Duan, W.S. and Lv, K.P. (2006) Application of the Homotopy Analysis Method to Solving Nonlinear Evolution Equations. Acta Physica Sinica, 55, 1555-1560.

[14] Zhao, X.S., Zhou, H.X., Tang, Y.N. and Jia, H.B. (2006) Travelling Wave Solutions for Modified Zakharov-Kuznetsov Equation. Applied Mathematics and Computation, 181, 634-648. https://doi.org/10.1016/j.amc.2006.01.049

[15] Wazwaz, A.M. (2008) The Extended Tanh Method for the Zakharov-Kuznetsov (ZK) Equation, the Modified ZK Equation, and Its Generalized Forms. Communications in Nonlinear Science and Numerical Simulation, 13, 1039-1047. https://doi.org/10.1016/j.cnsns.2006.10.007

[16] Tascan, F., Bekir, A. and Koparan, M. (2009) Travelling Wave Solutions of Nonlinear Evolution Equations by Using the First Integral Method. Communications in Nonlinear Science and Numerical Simulation, 14, 1810-1815. 
https://doi.org/10.1016/j.cnsns.2008.07.009

[17] Peng, Y.Z. (2009) Exact Travelling Wave Solutions for a Modified Zakharov-Kuznetsov Equation. Acta Physica Polonica A, 115, 609-612.

https://doi.org/10.12693/APhysPolA.115.609

[18] Noor, M.A., Mohyud-Din, S.T., Waheed, A. and Al-Said, E.A. (2010) Exp-Function Method for Traveling Wave Solutions of Nonlinear Evolution Equations. Applied Mathematics and Computation, 216, 477-483. https://doi.org/10.1016/j.amc.2010.01.042

[19] Naher, H. and Abdullah, F.A. (2012) The Improved (G'G)-Expansion Method for the (2+1)-Dimensional Modified Zakharov-Kuznetsov Equation. Journal of Applied Mathematics, 2012, Article ID: 438928. https://doi.org/10.1155/2012/438928

[20] Yuan, W.J., Huang, Y. and Shang, Y.D. (2013) All Traveling Wave Exact Solutions of Two Nonlinear Physical Models. Applied Mathematics and Computation, 219, 6212-6223. https://doi.org/10.1016/j.amc.2012.12.023 